\title{
Construcción de triángulos, recurso para impulsar un ambiente de indagación
}

Construction of Triangles, a Resource to Foster an Inquiry Environment

Artículo de investigación | Research article

Fecha de recepción: 23 de julio de 2018 Fecha de aceptación: 16 de mayo de 2019 Fecha de disponibilidad en línea: marzo de 2021

doi: 10.11144/Javeriana.m14.ctri

Eliana Martínez-Mora

emartinezm@pedagogica.edu.co instituto Pedagógico Nacional, Colombia D ORCID: https://orcid.org/0000-0003-0272-5286

Leonor Camargo-Uribe Icamargo@pedagogica.edu.co Universidad Pedagógica Nacional, Colombia ORCID: https://orcid.org/0000-0002-2237-7306

Para citar este artículo | To cite this article Martínez-Mora, E. \& Camargo-Uribe, L. (2021). Construcción de triángulos, recurso para impulsar un ambiente de indagación. magis, Revista Internacional de Investigación en Educación, 14,1-24. doi: 10.11144/Javeriana.m14.ctri 


\title{
Resumen
}

Este artículo de investigación presenta una vía para involucrar a estudiantes en ambientes de indagación en donde exploren, conjeturen, justifiquen y comuniquen sus hallazgos, al resolver problemas de construcción geométrica con apoyo de GeoGebra. Para ello, se empleó la metodología experimento de diseño, con estudiantes de 10 a 12 años, para poner la vía en funcionamiento y evaluar su efectividad. Se concluye que el reto de promover tal ambiente va más allá del diseño de situaciones problema. Se requiere un cambio en la cultura de la clase, promovido principalmente por el profesor, para generar un espacio colectivo de participación.

\section{Palabras clave}

Ambiente de la clase; aprendizaje; educación básica; geometría; igualdad de oportunidades

\begin{abstract}
This article provides a path to involve the students in environments for inquiry in which they will explore, conjecture, justify and communicate their findings, by solving problems of geometric construction aided by GeoGebra. We used the methodology of "experiment design" with 10-12 years old students in order to start up the path and evaluate how effective it is. We concluded that the challenge to Foster such environment goes beyond the problem situation design. It requires a change in the classroom culture, being fostered mostly by the teacher in order to create a collective participation space.
\end{abstract}

\section{Keywords}

Classroom environment; learning; equal opportunity; geometry; basic education 
Descripción del artículo | Article description

Este artículo de investigación reporta resultados del trabajo de grado Hacia un ambiente de indagación en una clase de geometría, realizado entre 2015 y 2016 por la primera autora como requisito parcial para optar al título de magíster en Docencia de las Matemáticas de la Universidad Pedagógica Nacional. La segunda autora fue la directora del trabajo.

\section{Introducción}

Este artículo es producto de una investigación que tenía como meta promover un ambiente de indagación en una clase de geometría de sexto grado de educación básica, involucrando a los estudiantes en la resolución colectiva de problemas de construcción geométrica, con el apoyo del programa de geometría dinámica GeoGebra. Ello implicó prestar atención al diseño de situaciones problema, la organización de la actividad matemática de los estudiantes, la gestión del profesor y las normas de la clase. En particular, nos enfocamos en promover prácticas matemáticas de explorar, conjeturar, argumentar y justificar y en prácticas sociales de comunicar, escuchar, disentir y acordar.

El estudio atendió algunas recomendaciones para Colombia del Ministerio de Educación (2006), relacionadas con los fines de la educación matemática que se dirigen a apoyar a los estudiantes en el desarrollo de procesos de razonamiento y comunicación. A lo largo de su escolaridad, los niños y jóvenes colombianos deben aprender a explorar, conjeturar y validar hechos matemáticos, comunicar e intercambiar ideas y trabajar colectivamente en pro de metas comunes. En ese sentido, el foco de la actividad matemática no es la memorización de información, procedimientos o algoritmos, sino la indagación matemática en ambientes de resolución de problemas que lleven a la apropiación significativa de los contenidos matemáticos del currículo.

La motivación inicial que derivó en la realización del estudio fue la toma de consciencia de que las clases de matemáticas dirigidas por la primera autora del artículo, en una institución educativa que se consideraba como centro de innovación e investigación, parecían estar muy poco permeadas por los planteamientos anteriores. Una reflexión preliminar acerca de la forma de gestionar sus clases puso de manifiesto que su enseñanza estaba regida bajo el paradigma del ejercicio (Cotton, 1998, citado por Skovsmose, 2000) y que los estudiantes estaban acostumbrados a este. En general, la enseñanza se hacía a partir de modelar frente a los estudiantes cómo se resolvían ejercicios propuestos en un texto guía y la ejercitación repetitiva de 
cálculos y algoritmos, aun en la clase de geometría. Este tipo de enseñanza hacía que los estudiantes dependieran de las indicaciones de la profesora para que ella les dijera qué tenían que hacer, buscaran la solución en un libro o la copiaran del cuaderno de algún compañero, sin un interés real por llevar a cabo una actividad matemática genuina; la mayoría esperaban a que la profesora aprobara sus respuestas sin buscar por sí mismos una forma de validarlas y tenían poco interés por las ideas de sus compañeros. Estas manifestaciones no son propias de un ambiente de indagación, y traían como consecuencia que el foco de atención en las clases se centrara en dominar algunos contenidos y procedimientos para lograr una buena calificación, pero no conducían a una actividad matemática que diera pie al desarrollo del razonamiento y la comunicación.

Diversos investigadores hacen un llamado a cambiar las prácticas propias del paradigma del ejercicio (e. g., Forman, 1996; Furinghetti, Olivero \& Paola, 2001; Goos, 2004; Quaranta \& Tarasow, 2004; Samper \& Molina, 2013; Yevdokimov, 2005) y promueven la búsqueda de experiencias escolares que permitan a los estudiantes experimentar ambientes de aprendizaje centrados en la resolución de problemas en los que niños y jóvenes tengan oportunidades de explorar, conjeturar, justificar, comunicar sus ideas y desarrollar un interés genuino por indagar.

A partir de tales llamados, nos propusimos diseñar, experimentar y evaluar (buscando indicios de conformación de un ambiente de indagación) una secuencia de enseñanza encaminada a promover un ambiente de indagación en la clase de geometría de estudiantes de 10 a 12 años. En este artículo presentamos los resultados de esta experiencia. La pregunta que orientó nuestro estudio fue "¿Qué aspectos de la interacción profesora-estudiantes hacen evidente el comienzo de la constitución de un ambiente de indagación?".

En busca de respuestas a esta pregunta, llevamos a cabo una investigación de diseño (Molina, Castro, Molina \& Castro, 2011) en la que decidimos aprovechar el papel mediador de GeoGebra para promover la indagación matemática (Goos, 2004; Richard, 1991). La metodología involucra la implementación de una secuencia de enseñanza que nosotras centramos en la construcción geométrica y en la gestión por parte de la profesora para impulsar la participación y la comunicación como motores de la indagación. En las siguientes secciones, presentamos el marco teórico que orientó la investigación, el diseño metodológico implementado y algunos análisis de los resultados obtenidos. Esperamos suscitar una reflexión sobre las posibilidades reales de incentivar un ambiente de indagación y sobre el papel determinante de la cultura de la clase para que los estudiantes asuman un papel activo y acojan y respalden las normas que promueven una comunidad de indagación. 


\section{Referentes conceptuales}

El marco de referencia del estudio se centra en fundamentar una postura sobre qué entendemos por ambiente de indagación. Esto con el fin de tener herramientas analíticas para analizar el efecto del experimento de diseño sobre su constitución.

Nuestra conceptualización de ambiente de indagación toma elementos de diversas fuentes. En primer lugar, de la caracterización que hacen Richards (1991), Puentes (2015) y Goos (2004) de las clases, como comunidades de indagación (los primeros dos) o de investigación (la tercera), que se implementan a partir de acciones del profesor y de los estudiantes, con las cuales ellos pueden participar en discusiones matemáticas, actuar matemáticamente, solucionar problemas, formular conjeturas y escuchar y contrastar argumentos matemáticos. En segundo lugar, del planteamiento que hacen Quaranta \& Tarasow (2004) de una comunidad de validadores que busca impulsar actividades para que los estudiantes encuentren criterios para validar sus propias producciones. En tercer lugar, de la sugerencia de Furinghetti et al. (2001) de involucrar a los estudiantes en la resolución de problemas abiertos, en los que haya posibilidad de usar diferentes estrategias de solución que den lugar a diversas conjeturas y justificaciones. Y, en cuarto lugar, de la propuesta de Forman (1996), quien menciona que en una comunidad de indagación se tiene en cuenta la importancia de cada uno de los participantes, se impulsa el trabajo colaborativo, se promueve el contraste de ideas y la argumentación, y los significados se construyen de manera negociada.

Con base en la revisión de la literatura, conceptualizamos un ambiente de indagación en la clase de matemáticas. Entendemos este como una atmósfera promovida por un conjunto de prácticas de enseñanza y de aprendizaje que propician un involucramiento activo de los estudiantes en la actividad matemática y en la que ellos asumen un papel protagónico en la construcción de conocimiento, en el establecimiento de normas de clase, en la toma de decisiones y asumen un compromiso genuino por resolver las inquietudes que surgen en el aula, en conjunto con los compañeros y con el profesor.

Al referirnos a las normas de clase, distinguimos, como lo sugieren Yackel \& Cobb (1996), entre los acuerdos que los miembros de una comunidad establecen para tener unos adecuados canales de comunicación (normas sociales) y los acuerdos que establece el actuar matemático en clase (normas sociomatemáticas). Ambos tipos de normas son determinantes en la constitución de un ambiente de indagación. Las primeras regulan los turnos de la palabra, la escucha, etc. Las segundas, regulan lo que el grupo acepta como conjetura y justificación, por ejemplo. 
Como lo señalan diversos investigadores (e. g., Camargo \& Sandoval, 2017; Laborde, 2000; Mariotti, 2000), una estrategia para lograr el involucramiento activo de los estudiantes en la actividad matemática consiste en darles la oportunidad de hacer uso de un programa informático de geometría dinámica para resolver problemas. Particularmente en geometría, las opciones de representación y manipulación de elementos representados generan ricas opciones para impulsar procesos de exploración, conjeturación y justificación, y permiten a los estudiantes rebasar el universo perceptual en el que usualmente se ubican para acercarse al estudio de las propiedades geométricas. Por esto consideramos que el uso de programas, como GeoGebra, contribuye a generar un ambiente de indagación.

\section{Metodología}

Con la intención de promover el surgimiento de un ambiente de indagación llevamos a cabo una investigación de diseño. Como señalan Molina et al. (2011), esta metodología "persigue comprender y mejorar la realidad educativa a través de la consideración de contexto naturales en toda su complejidad y del desarrollo de un análisis paralelo de un diseño instruccional específico" (p. 75). Generalmente, el profesor del curso en el que se hace la experimentación hace parte del equipo de investigación. Desde la óptica de esta metodología, su participación "enriquece la elaboración y análisis del diseño, así como la recogida e interpretación de los datos, e incrementa la calidad del proceso de investigación" (Molina et al., 2011, p. 77). El experimento fue llevado a cabo en un curso de geometría con 33 estudiantes de grado sexto. El grupo fue escogido porque, a pesar de estar acostumbrado al ambiente generado por el paradigma del ejercicio, la mayoría de los estudiantes estaban interesados en la clase de geometría, eran responsables académicamente y se mostraban curiosos intelectualmente. Además, existía muy buena relación entre los estudiantes y la profesora. Estos elementos nos permitían suponer que la marcha del experimento no se vería afectada negativamente por factores ajenos al diseño propuesto.

Planeamos una secuencia de enseñanza con las siguientes características, que, desde nuestro punto de vista y con base en la información obtenida en la revisión de literatura, podrían comenzar a promover un ambiente de indagación en clase de geometría:

- Los estudiantes son invitados a buscar soluciones a situaciones problema de construcción geométrica con el apoyo de un programa de geometría dinámica, que brinda a los estudiantes un entorno enriquecido para impulsar la exploración, la conjeturación y la justificación. 
- La profesora organiza la clase contemplando dos momentos. Primero, los estudiantes trabajan en parejas o tríos alrededor de las situaciones propuestas. Segundo, las soluciones se discuten en una puesta en común, en la que la profesora guía una discusión tendiente a aprobar o desaprobarlas colectivamente.

- La profesora promueve la participación de los estudiantes tanto en el trabajo en parejas o tríos como en la plenaria e impulsa la expresión y escucha de ideas. Más que aprobar o desaprobar intervenciones, busca que los estudiantes tomen una postura sobre las propuestas de solución y validen las producciones que consideren correctas. La pregunta que reina en el ambiente es el porqué de estrategias, soluciones, posturas, afirmaciones, etc.

- Profesora y estudiantes velan por el establecimiento de normas sociales y sociomatemáticas (Yackel \& Cobb, 1996) que favorezcan la expresión y escucha de puntos de vista, estrategias, soluciones, argumentos y acuerdos.

Decidimos enfocar la secuencia de enseñanza en problemas que invitaban a la construcción de triángulos isósceles y equiláteros, proponiendo diversas estrategias que se valían de herramientas geométricas, como circunferencias y mediatrices. Propusimos tres problemas introductorios y seis problemas retadores (tabla 1).

Tabla 1

Situaciones problema introductorias y retadoras

\section{Problemas introductorios}

P1: Construir un punto $A$. Construir varios puntos que estén a la misma distancia de $A$.

P2: Construir varios puntos alineados.

P3: Construir dos segmentos congruentes.

\section{Problemas retadores}

P4: Dados dos puntos $A$ y $B$, construir un punto $C$, tal que $B$ sea el punto medio del segmento $A C$.

P5: Construir un triángulo isósceles $A B C$.

P6: Construir un segmento $A B$. Construir un triángulo isósceles $A B C$, tal que el lado $A B$ no sea congruente con ninguno de los otros lados.

P7: Construir un triángulo equilátero usando solo una circunferencia.

P8: Dada la recta $A B$ y un punto $C$ que no pertenece a la recta $A B$, construir un punto $D$, tal que esté a un lado distinto de $C$ con respecto a la recta y que para cualquier punto $X$ de la recta $A X=X D$.

P9: Construir un triángulo y sus mediatrices. Encontrar una propiedad que caracterice las mediatrices y justificarla. 
Los problemas introductorios tenían como objetivo que los estudiantes comenzaran a usar el programa GeoGebra, identificaran las herramientas básicas de construcción geométrica, interpretaran los íconos que informan cómo se usa cada herramienta y empezaran a usar la función de "arrastre" (Laborde, 2000) para distinguir entre una construcción blanda (basada en la percepción visual, cuyas propiedades se pierden al arrastrar los elementos libres de la construcción) y una robusta (basada en construcciones auxiliares, cuyas propiedades geométricas se mantienen invariantes al arrastrar los elementos libres). También pretendíamos que institucionalizaran hechos geométricos como las definiciones de circunferencia, segmentos congruentes y radio de una circunferencia, así como la propiedad de congruencia de los radios de una circunferencia. Pero más que aprender tales hechos, buscábamos que los identificaran como herramientas de construcción y verificación de equidistancia entre puntos, alineación de puntos y congruencia de segmentos.

Los problemas retadores buscaban que los estudiantes se involucraran en un ambiente de indagación al asumir el desafío de buscar soluciones a cada uno, explorando posibilidades e identificando posibles herramientas de construcción. Pretendíamos, además, que propusieran y justificaran conjeturas sobre cómo lograr la construcción solicitada. Para justificar, deberían valerse de hechos geométricos ya institucionalizados.

La aplicación de la secuencia de enseñanza se hizo en nueve sesiones de clase de 90 minutos. La profesora, primera autora de este artículo, dirigió la clase intentando promover un ambiente de indagación. En las clases, además de ella, estaban dos auxiliares de investigación, que videogrababan el trabajo hecho por cada pareja y las puestas en común. En reuniones posteriores a cada clase, los miembros del equipo de investigación discutían sobre los aciertos y desaciertos de la sesión y sugerían a la profesora ideas para la gestión de la siguiente clase.

Terminada la implementación, la profesora revisó varias veces cada uno de los videos de las sesiones de clase en busca de evidencias de un ambiente de indagación. A partir de la sensibilización lograda gracias al marco teórico construido previamente, ella escogió 25 fragmentos de clase que consideró representativos del ambiente logrado. Después, el equipo de investigación desarrolló un ejercicio de codificación emergente, pero informado a partir de la revisión de la literatura, de las interacciones de cada fragmento, señalando oraciones, frases, expresiones o conjuntos de intervenciones que consideraban indicadores de un ambiente de indagación e ideando un código para cada indicador (la nomenclatura usada comienza con E y $\mathrm{P}$ para distinguir entre acciones de los estudiantes o de la profesora y luego tiene tres letras mayúsculas, que corresponden a letras iniciales de 
palabras centrales en la descripción). La descripción de cada código y su categorización fue emergiendo a medida que iban construyendo el listado de códigos y agrupando en un código información similar (tablas 2 y 3).

Tabla 2

Códigos para las acciones de los estudiantes

\begin{tabular}{|c|c|c|}
\hline \multirow{3}{*}{ Argumentación } & E-APE & $\begin{array}{l}\text { Argumenta su postura empíricamente, intentando convencer a sus compañeros o } \\
\text { a la profesora de que lo que dice es cierto, con base en construcciones, ejemplos, } \\
\text { medidas, etc. }\end{array}$ \\
\hline & E-APT & $\begin{array}{l}\text { Argumenta su postura teóricamente, intentando convencer a sus compañeros } \\
\text { o a la profesora de que lo que dice es cierto, con base en hechos geométricos } \\
\text { conocidos por el grupo. }\end{array}$ \\
\hline & $\mathrm{E}-\mathrm{PQI}$ & $\begin{array}{l}\text { Pregunta por qué, para incentivar la argumentación o la expresión coherente, } \\
\text { clara y completa de las ideas. }\end{array}$ \\
\hline \multirow{3}{*}{ Toma de postura } & E-EXP & $\begin{array}{l}\text { Explica su punto de vista para hacerse entender, es decir, busca que sus } \\
\text { compañeros comprendan algo que ha dicho introduciendo el lenguaje acordado. }\end{array}$ \\
\hline & E-TP & $\begin{array}{l}\text { Toma postura acerca de una propuesta solución, manifestando acuerdo o } \\
\text { desacuerdo acerca de una idea, estrategia, solución a un problema, propuesta } \\
\text { por un compañero o la profesora. }\end{array}$ \\
\hline & E-RA & Refuta un argumento expresado por un compañero o por la profesora. \\
\hline \multirow[t]{2}{*}{ Responsabilidad } & E-APA & $\begin{array}{l}\text { Propone realizar una acción para aclarar o verificar la estrategia de solución } \\
\text { propuesta por un compañero. }\end{array}$ \\
\hline & E-RN & Contribuye a regular una norma para favorecer un clima de comunicación. \\
\hline
\end{tabular}

Fuente: elaboración propia

Tabla 3

Códigos para las acciones del profesor

\begin{tabular}{|c|c|c|}
\hline \multirow[b]{2}{*}{ Argumentación } & P-PQA & Pregunta por qué para solicitar argumentos sobre ciertas posturas o afirmaciones. \\
\hline & P-PQC & $\begin{array}{l}\text { Pregunta por qué en busca de profundizar en la argumentación y que los } \\
\text { estudiantes sean específicos y claros en la toma de postura. }\end{array}$ \\
\hline \multirow{2}{*}{ Toma de postura } & P-OEA & Orienta y media el establecimiento de acuerdos matemáticos. \\
\hline & P-VAI & Valora explícitamente los aportes individuales para promover la expresión de ideas. \\
\hline \multirow{5}{*}{ Confrontación } & P-PPV & Problematiza el punto de vista de algún estudiante. \\
\hline & P-PCP & $\begin{array}{l}\text { Promueve la confrontación de posturas mediando en el establecimiento de } \\
\text { acuerdos matemáticos. }\end{array}$ \\
\hline & P-PEl & Promueve la escucha de ideas para favorecer el intercambio comunicativo. \\
\hline & P-UT & $\begin{array}{l}\text { Orienta el uso de términos matemáticos o expresiones con las cuales se expresen } \\
\text { las ideas de manera más clara y sintética. }\end{array}$ \\
\hline & P-AT & $\begin{array}{l}\text { Aclara términos o da la oportunidad de que algunos estudiantes aclaren algunos } \\
\text { términos especializados. }\end{array}$ \\
\hline \multirow{3}{*}{ Responsabilidad } & P-APO & $\begin{array}{l}\text { Admite que su planteamiento sea objetado, manteniendo la disposición de } \\
\text { escucha frente a la toma de postura que adoptan los estudiantes con respecto a } \\
\text { lo que ha dicho. }\end{array}$ \\
\hline & P-CRV & $\begin{array}{l}\text { Cede la responsabilidad de validación a los estudiantes, impulsando el } \\
\text { establecimiento de criterios para validar sus respuestas o las de los demás. }\end{array}$ \\
\hline & P-AIS & Ahonda en lo dicho por un estudiante para indagar por el significado. \\
\hline
\end{tabular}


Después de analizar los 25 fragmentos, seleccionamos 10 en los que encontramos mayores indicios de un comienzo de ambiente de indagación. Para cada uno de ellos elaboramos una narración, tipo viñeta, que da cuenta de los sucesos de la clase y de la interpretación que hacemos relacionada con la posible configuración de un ambiente de indagación.

\section{Análisis de resultados}

En esta sección ejemplificamos los análisis con cuatro narraciones que ilustran la interacción en clase relacionada con las situaciones problema P5 y P6, pues queremos enfocarnos en la construcción de triángulos, en el marco de la secuencia de enseñanza.

\section{Narración 1}

La profesora propone resolver la situación problema P5: construir un triángulo isósceles usando GeoGebra. Mientras los estudiantes trabajan en parejas, ella se acerca a los grupos y les pide comentar sus propuestas de solución. Por ejemplo, dialoga con Camilo y Edwin, quienes tienen la figura 1 en la pantalla y le muestran entusiastas lo que han hecho, esperando probablemente una evaluación positiva.

Figura 1

Construcción de triángulo isósceles

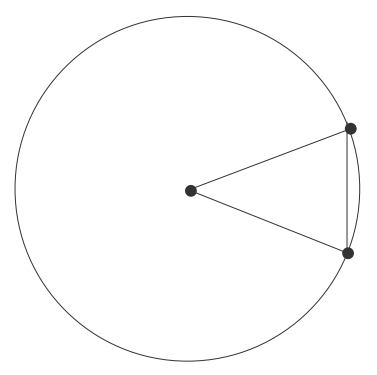

Fuente: elaboración propia

1. Profesora: Bueno, ¿qué pasó ahí?, ¿y por qué [construyeron una] circunferencia?... (Señala al grupo de al lado conformado por Andrés y Juan).

2. Camilo: iEllos usaron circunferencia!

3. Profesora: iAh! ¿O sea que te copiaste?

4. Camilo: Pues... yo dije... vamos a usar esa forma. Como tú los dejaste a ellos (Mira a Andrés y Juan). 
La profesora les pide justificar el procedimiento usado [1], invitándolos a que elaboren una argumentación [P-PQA ]. Camilo explica que él y su compañero usaron el mismo procedimiento que otro grupo $[2,4]$. La profesora lo interroga [3], promoviendo que sea específico y claro en la toma de postura asumida $\left[\mathrm{P}-\mathrm{PQC}_{1}\right]$. Andrés interviene en la conversación: "¿Sí ves, profe? Se copiaron y no saben por qué" [5]. Con su intervención parece retar a sus compañeros a explicar la construcción [E-PQI ]. La profesora se dirige a Camilo: "Bueno, ¿por qué usaste una circunferencia?, ipor qué aparece la circunferencia?" [6]. Pretende que explique el procedimiento $\left[\mathrm{P}-\mathrm{PQA}_{2}\right]$. Camilo propone una explicación, que muestra que, si bien se valió de lo que vio hacer a sus compañeros, entiende para qué se hace:

7. Camilo: Porque del punto centro (señala el centro de la circunferencia) ya hay una medida a acá (señala el extremo del segmento que usó como lado del triángulo) y de aquí (señala el centro de la circunferencia) a aquí (señala el extremo del otro lado). Entonces, si teníamos la misma medida de acá a acá (señala los dos segmentos), tenemos dos lados congruentes. Entonces este [segmento] no tiene la misma medida que estos dos lados (señala la cuerda que no es radio). Y ahí se forma un triángulo isósceles.

8. Profesora: ¿Por qué esos dos lados son congruentes?

Camilo elabora un argumento empírico [7] [E-APE ]. La profesora no queda satisfecha y le pide justificar por qué afirma que dos lados del triángulo son congruentes [8] [P-PQA $]$ ]. Con ello promueve la elaboración de argumentos teóricos $\left[\mathrm{P}-\mathrm{PQC}_{2}\right]$. Espera que el estudiante aluda a la propiedad de la congruencia de los radios de la circunferencia, institucionalizada al resolver el problema P2. Su pregunta conduce al siguiente diálogo:

9. Camilo: Porque tienen la misma medida.

10. Profesora: ¿Por qué tienen la misma medida?

11. Camilo: Porque tienen la misma medida del centro aquí (señala el extremo de un lado) y del centro a aquí (señala el otro extremo).

12. Profesora: ¿Y qué te permite decir que son congruentes? O sea ¿qué figura, o qué usaste para decir que son congruentes?

13. Camilo: Un círculo.

14. Profesora: ¿Un círculo?

15. Camilo: Bueno, una circunferencia.

Camilo repite el argumento refiriéndose a que los segmentos miden lo mismo [9] [E-APE ${ }_{2}$. Ante la insistencia de la profesora [10] [P-PQA 4 ], amplía su explicación, aludiendo implícitamente a la equidistancia de los puntos de 
la circunferencia al centro, y por lo tanto consideramos que elabora un argumento teórico [11] [E-APT ]. La profesora busca que aclare la explicación, quizás mencionando explícitamente la definición de circunferencia como garantía [12] [P-PQC $]$.

Desde nuestro punto de vista, el fragmento de interacción es un indicio de conformación de un ambiente de indagación. En primer lugar, porque los estudiantes no esperan que sea la profesora quien les diga cómo construir un triángulo isósceles, sino que ellos tienen que encontrar y justificar una vía para hacerlo. En ese sentido, aunque en esta pareja de estudiantes no tuvo lugar una real búsqueda de una estrategia de solución, los estudiantes estaban resolviendo una situación problema (Furinghetti et al., 2001) y debían formular, a manera de conjetura, cómo hacer la construcción (Yevdokimov, 2005). Camilo y Edwin optaron por la vía del menor esfuerzo, y decidieron reproducir el procedimiento de sus compañeros Andrés y Juan. En un ambiente de indagación esta acción no es censurada, sino permitida, pues se admite la colaboración de los compañeros (Forman, 1996). En segundo lugar, porque la profesora y el estudiante Andrés promueven la argumentación para validar el procedimiento (Quaranta \& Tarasow, 2004). Destacamos la intervención de Andrés [5], porque espontáneamente contribuye a establecer la norma de justificar lo que se hace o se afirma.

Visto en retrospectiva, hay varias acciones que la profesora pudo llevar a cabo en aras de una mejor gestión de un ambiente de indagación. En primer lugar, hubiera podido ahondar en las razones por las que a Andrés se le ocurrió usar una circunferencia y a Camilo le pareció oportuno usar ese procedimiento. Esto haría que Andrés tuviera la oportunidad de exponer su planteamiento y no se viera como si la estrategia surgiera por adivinación. En segundo lugar, la profesora hubiera podido involucrar a Edwin y a Juan en la conversación, preguntándoles si entendían y estaban de acuerdo con la estrategia, si podían reproducirla, si sabían para qué se hacía cada construcción y si podían explicarla a otros compañeros. En tercer lugar, si ella quería que Camilo o Andrés propusieran un argumento teórico, en lugar de preguntarles por qué los lados del triángulo tenían la misma medida o en cuál figura se habían apoyado, hubiera podido pedirles que explicaran por qué haciendo la construcción como la explicaron podían afirmar que las medidas de los lados eran iguales. Esta gestión requiere una experticia en la profesora que ella estaba hasta ahora ganando.

\section{Narración 2}

En la plenaria, la profesora decide poner en discusión la estrategia usada por Fabián y Antonio. Ellos pasan al frente y reproducen la construcción. Construyen tres puntos, $A, B$ y $C$, y los segmentos $A B, A C$ y $B C$. 
Después arrastran los vértices del triángulo hasta que perceptualmente dos lados parecen congruentes y luego usan la función candado de GeoGebra, que fija los puntos y no permite el arrastre. Esta función no había sido trabajada previamente en clase. Tan pronto terminan de presentar la construcción, Carolina dice, en tono irónico: "Y ahora ellos dijeron que ese es un triángulo isósceles" [16], manifestando con ello su desacuerdo con la estrategia [E-TP $]$. Camilo pide pasar al frente [17], probablemente para comprobar si la construcción era blanda o robusta, también manifestando poca credibilidad con el procedimiento $\left[\mathrm{E}-\mathrm{TP}_{2}\right]$, pero, además, sugiriendo una estrategia para validarla [E-APA $]$. Algunos estudiantes también quieren hacer la comprobación, y la profesora pide a Edwin que lo haga. Él intenta, sin lograrlo, arrastrar los puntos. Esto provoca el siguiente diálogo:

18. Margarita: iProfe! Puede que ahí en ese momento esté bien, pero, para que esté bien, el punto $B$ se tiene que mover, a ver si la congruencia se queda o se va.

19. Camilo: $\quad$ Pues le quitas el candadito al punto $B$ (Mira a Margarita).

20. Margarita: Pero igual está mal hecho, porque eso sería hacer trampa.

21. Varios: iSí!

22. Varios: iNo!

23. Lorena: No, porque nunca dijeron que no se podía [usar] esa opción.

24. Margarita: Pero eso no tiene nada que ver.

Margarita expresa su desacuerdo con la estrategia [E-TP $]$ y explica su punto de vista [18] [E-EXP $]$. Para ella, la forma en que han procedido Fabián y Antonio es una trampa [20]. Algunos compañeros respaldan su punto de vista y otros lo rechazan $[21,22]\left[\mathrm{E}_{-} \mathrm{TP}_{4}\right]$. Lorena refuta el argumento de Margarita [23] [E-RA, , aludiendo a que el uso de la función candado de GeoGebra no estaba prohibida. Margarita intenta explicar su punto de vista, que va más allá de la prohibición o no del uso de una función [24] [E-EXP $\mathrm{F}_{2}$. La profesora interviene preguntando: "¿Entonces qué le decimos al compañero?, ¿qué es lo que debe mantenerse?" [25] [P-OEA $]$. Carolina aclara que hay que verificar si la congruencia se mantiene cuando arrastran elementos de la construcción [26] [E-APA $A_{2}$. La profesora media en el establecimiento de un acuerdo [26] [P-OEA ${ }_{2}$ :

26. Profesora: Bueno. Entonces, ¿vamos a poder usar "candadito" en otras construcciones?

27. Varios: iNo!

28. Profesora: La idea es que, en este tipo de construcciones, se pueda

29. Mateo: iMover! 
En la narración se hacen evidentes varios rasgos de inicio de un ambiente de indagación. Principalmente, al poner en discusión una estrategia que fija la construcción e impide modificar la posición de los puntos en la construcción, la profesora promueve la toma de postura frente a la estrategia, promoviendo que algunos estudiantes manifiesten explícitamente su acuerdo o desacuerdo, expliquen su punto de vista, propongan acciones de verificación y refuten el de sus compañeros. Es decir, los estudiantes asumen la responsabilidad de la validación (Quaranta \& Tarasow, 2004) y hacen aportes valiosos de acuerdo con su experiencia y conocimiento (Forman, 1996). Margarita y Camilo desempeñan papeles centrales al promover la discusión espontáneamente y dar ideas para aceptar o rechazar la propuesta. La profesora no asume un papel de autoridad. Sus intervenciones buscan que los estudiantes aclaren sus posturas y median en el establecimiento de una norma sociomatemática: la función candado no puede emplearse porque las construcciones deben ser dinámicas.

Visto en retrospectiva, hubiera sido deseable que ella pidiera a Carolina y a Lorena ampliar su punto de vista para que quedara más claro y que la norma matemática hubiera sido formulada totalmente por los estudiantes. También podría haber preguntado a otros estudiantes si entendían la dificultad de la propuesta de Juan y Antonio, pues ella presupuso que todos entendían. En ese sentido, podría haber limitado un poco más su aporte y mediado la comunicación para hacerla más efectiva y propiciar una mejor negociación de significados.

\section{Narración 3}

La profesora propone a los estudiantes el problema P6: Construir un segmento $A B$. Luego, construir un triángulo isósceles $A B C$, tal que $A C=B C$. A diferencia de la resolución del problema P5, en la que la mayoría de las parejas encontraron rápidamente un procedimiento de construcción, al enfrentar el problema P6 los estudiantes no saben cómo proceder. No encuentran la forma de realizar la construcción pedida y se percibe desánimo. Al ver que no se les ocurre una vía, la profesora les dice que miren la posibilidad de construir dos circunferencias. Después de un tiempo, interactúa con algunos grupos. Veamos apartes del diálogo que sostiene con Margarita y Carolina. Ellas tienen en la pantalla una construcción como la figura 2(a). La profesora les pide que le expliquen cómo hicieron la construcción, por lo que ellas hacen visibles los objetos ocultos (figura 2[b]). Margarita comienza la explicación: 
Figura 2

Propuesta de construcción de Margarita y Carolina

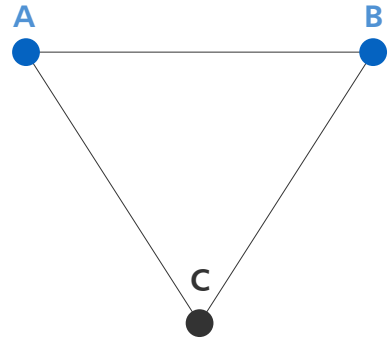

(a)

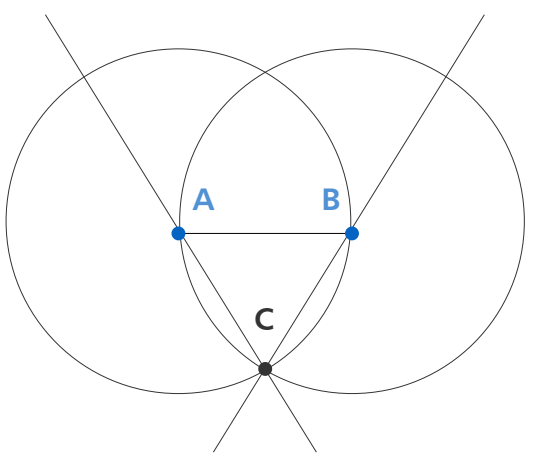

(b)

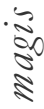

15

Fuente: elaboración propia

30. Margarita: Pues yo... Pues, cuando dijiste "dos circunferencias", lo primero que se me vino a la cabeza, era hacer el segmento (señala el segmento AB) y comenzar por acá (señala el punto A) y que terminara acá (señala el punto B). O sea que este fuera el... (señala el punto A).

31. Profesora: Circunferencia con centro ien?

32. Margarita: En [el punto] $A$.

33. Profesora: ¿Y radio?

34. Margarita: En [el punto] $B$. iAh, no! Perdón. Radio $A B$ (señala el segmento $A B$ ). Y luego hacer la otra circunferencia que comience en el centro $B$, para que, al intersecarse entre las dos circunferencias, ahí sería el punto de intersección. Por eso está de otro color el punto C. Ya que siendo este el punto de intersección (/o señala) no podrá moverse para ningún lado y se queda la congruencia.

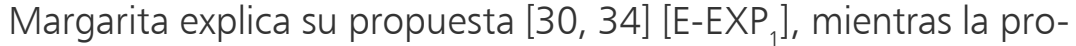
fesora la orienta en el uso de términos apropiados [31, 33] [P-UT $]$. Después, la profesora le pide justificar por qué son congruentes [35] $\left[\mathrm{P}-\mathrm{PQA} \mathrm{A}_{1}\right.$ :

36. Margarita: Por la circunferencia (señala el segmento AB). O sea, estos dos segmentos (segmento $A C$ y segmento $B C$ ) tienen la misma medida al conectarse en el punto $C$ y este (segmento $\mathrm{AB}$ ) no. O sea, si tú ves en este circulito (señala el punto de intersección C) en donde está el punto C.

37. Alejandra: ¿Cuál circulito?

38. Margarita: Bueno, la mitad de las dos circunferencias. 
Margarita elabora una argumentación empírica para justificar por qué los segmentos $A C$ y $B C$ son congruentes [36] [E-APE $]$, aunque realmente no hace explícito que se trata de dos radios de circunferencias congruentes. Alejandra, una estudiante del grupo de al lado, que está atenta a la explicación, interviene para pedir una aclaración [37] [E-PQI ], indicando que se interesa por las ideas de su compañera. Con su intervención, contribuye a regular la norma de expresar claramente las ideas [38] [E-RN ${ }_{1}$.

La profesora, más que fijarse en el argumento dado por Margarita, centra la atención en la afirmación que ella hace, en la que dice que el segmento $A B$ no es congruente con los segmentos $A C$ y $B C$. Les pregunta: " $i Y$ esos dos (segmentos AB y AC) son congruentes?" [39]. Margarita responde que no [40] y Carolina dice que sí [41], y muestra a su compañera los segmentos $A B$ y $A C$, pero ella no se convence. Cuando la profesora les pide justificar por qué sí o por qué no promueve la confrontación de posturas $\left[\mathrm{P}-\mathrm{PCP} \mathrm{P}_{1}\right]$ e intenta que lleguen a un acuerdo [P-OEA $]$, pero las estudiantes no responden. Entonces ella decide colorear las circunferencias y orientar la visualización hacia los lados del triángulo como radios de cada una de ellas (figura 3).

Figura 3

Estrategia de la profesora para favorecer la argumentación

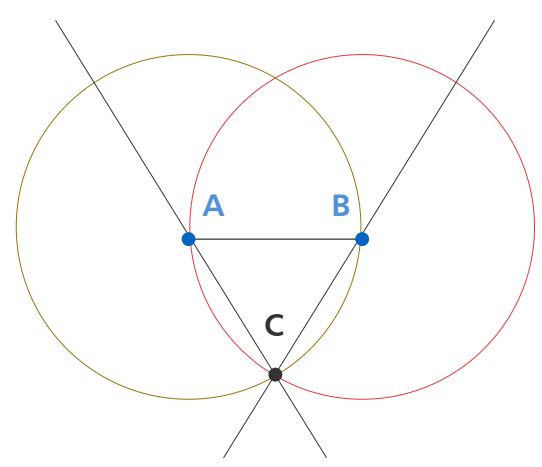

Fuente: elaboración propia

En lugar de dar a las estudiantes la justificación sobre la congruencia de los tres lados del triángulo, la profesora intenta que identifiquen un criterio que les permita dirimir la diferencia de opiniones: “¿Por qué no son congruentes? [...]. Bueno. De la circunferencia roja (con centro en B) ¿cuáles son los radios?" [39] [P-CRV $]$ ]. Carolina sigue el razonamiento y propone un argumento empírico: "Mirando la circunferencia roja, (los segmentos) $A B$ y $B C$ los unimos y son congruentes (señala en la pantalla el punto A y luego dirige 
el dedo hacia el punto C)" [40]. [E-APE ${ }_{2}$. La estudiante parece evocar la propiedad de los radios de una circunferencia, aunque no alude a ella explícitamente. Margarita no queda convencida y manifiesta su desacuerdo: "No, no son congruentes" [41] [E-TP $]$, mostrando con ello que no está dispuesta a aceptar que su compañera tiene la razón, hasta no entender por qué.

Afirmamos que en el diálogo hay elementos que son un germen de ambiente de indagación. Se presentan opiniones encontradas y la profesora intenta que las estudiantes encuentren una vía de validación, orientando el establecimiento de acuerdos (Forman, 1996; Goos, 2004). Las estudiantes asumen la responsabilidad de la validación y no se limitan a esperar la confirmación por parte de la profesora (Quaranta \& Tarasow, 2004). Sin embargo, la situación podría haberse explotado de manera más conveniente si la profesora hubiera pedido a Margarita interpretar lo dicho por Carolina y, después de haberse asegurado de que ella había comprendido lo que decía su compañera, le hubiera pedido tomar una postura al respecto.

Margarita no parece seguir la idea que se está poniendo en juego. La profesora la interroga al respecto [42], y ella expresa: "Yo no veo nada de la congruencia" [43]. Nuevamente, manifiesta su desacuerdo con lo dicho por la profesora y por Carolina [E-TP ${ }_{2}$. La profesora le muestra los segmentos $A B$ y $A C$ y le pide responder si son radios de una circunferencia y decir por qué son congruentes [44] [P-PQA $]$. Finalmente, Margarita afirma que sí lo son [45] [E-TP $]$ y argumenta empíricamente: "Porque comienzan en el mismo (señala el punto $A$ ), tienen intersección en el centro $(A)$ y los puntos $B$ y $C$ están acá (señala la circunferencia), en la circunferencia" [45] $\left[\mathrm{E}-\mathrm{APE}_{3}\right]$. Mediante un razonamiento similar, concluyen que los lados $B A$ y $B C$ son congruentes.

La profesora sintetiza el razonamiento: "Entonces, miren lo que han dicho: que $A B$ y $A C$ son congruentes, pero también me han dicho que $A B$ y $B C$ son congruentes ¿Qué podemos concluir?" [46] [P-UT 2 ]. Ella pretende que la discusión sobre la congruencia de los tres lados del triángulo quede dirimida al comparar las dos conclusiones. Sin embargo, Carolina rechaza el procedimiento de construcción que ella y Margarita propusieron "No es un triángulo isósceles" [47] [E-TP 4 ]. Margarita pregunta "¿Por qué no?" [48] $\left[\mathrm{E}-\mathrm{PQI}_{2}\right]$, y Carolina explica "Porque todos tienen los lados iguales y entonces sería un polígono regular. Digo, un triángulo, eh, iequilátero!" [49] [E-EXP ${ }_{2}$.

Carolina y Margarita exhiben una actitud de indagación genuina. Carolina invalida su propia propuesta de construcción y, por solicitud de su compañera, da una explicación a su punto de vista. No se trata simplemente de ir respondiendo las preguntas de la profesora. Carolina toma la iniciativa y se responsabiliza de la validación, de acuerdo con su experiencia y conocimiento (Forman, 1996; Goos, 2004; Quaranta \& Tarasow, 2004). 
La intervención de Carolina es aprovechada por la profesora para incentivar una discusión: "¿Un triángulo equilátero no es isósceles?" [50] [P$\mathrm{PCP}_{2}$ ]. Nuevamente, hay posturas encontradas. Esta vez Carolina afirma que no y Margarita, que sí. La profesora pregunta por qué [P-PQA $]$, y ambas estudiantes elaboran argumentos explicando su punto de vista $\left[\mathrm{E}-\mathrm{EXP}_{3}\right]$.

54. Carolina: No, no, no, no. Porque el isósceles tiene solo un lado que no es igual, pero, o sea...

55. Margarita: Pues yo diría que puede ser, porque, pues, tener el triángulo equilátero, pues puedes modificar la base para que se convierta en un isósceles.

La profesora problematiza el punto de vista de las estudiantes [P-PPV ${ }_{1}$, buscando que sean específicas y claras en la toma de postura [P-AIS $]$. Primero, pide a las estudiantes explicitar qué entienden por triángulo isósceles y por triángulo equilátero [56] [P-PQC $]$ y luego busca que relacionen las dos definiciones. Margarita dice que el triángulo isósceles tiene solo dos lados de igual medida y Carolina acepta que un triángulo equilátero puede ser isósceles [64], aunque no explica por qué. Ella además regula la norma de expresarse correctamente para favorecer la comunicación: "No se dice de igual medida, se dice congruentes" [59] [E-RN 2 ]. Las estudiantes descartan el procedimiento de construcción porque quieren obtener un triángulo isósceles no equilátero. Ningún grupo logra esa construcción.

\section{Narración 4}

En la plenaria, la profesora decide explicar el procedimiento de construcción introduciendo la herramienta compás de GeoGebra para construir un triángulo isósceles $A B C$, tal que el lado $A B$, que se construye inicialmente, no sea congruente con los otros dos lados. El hecho de que ella haga una explicación al grupo no es ajeno a un ambiente de indagación. Como indica Forman (1996), como miembro experto, ella tiene la responsabilidad de brindar información que no está a disposición de los estudiantes, cuando se requiera.

Los estudiantes están atentos, pues quieren saber cómo se hace la construcción. El hecho de haber intentado hacerla y no haber podido genera en ellos un interés genuino por la explicación de la profesora. Incluso, algunos de ellos piden a otros que guarden silencio para poder escuchar, contribuyendo a regular una norma para favorecer la comunicación [E-RN $N_{1}$.

Al momento de hacer la explicación, la profesora inicialmente se confunde, equivoca el procedimiento y no logra la construcción deseada en dos ocasiones. Los estudiantes manifiestan su desacuerdo con lo que ella hace $\left[\mathrm{E}_{-T P_{1}}\right]$. Ella mantiene una actitud de escucha admitiendo las 
objeciones de los estudiantes [P-APO, ]. Finalmente, hace una circunferencia con centro en $A$ y un segmento control para definir el radio. Margarita, Carolina y Camilo captan el procedimiento y la apoyan en la realización de la construcción. Finalmente, obtienen una construcción como la de la figura 4.

Figura 4

Construcción de triángulo isósceles hecha por la profesora

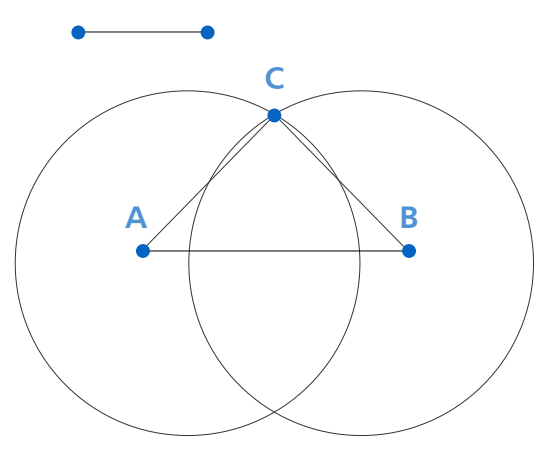
aunque podría parecer contraproducente que el profesor no tenga el control total de la situación, desde el punto de vista de la generación de un ambiente de indagación es factible asumir el hecho como una situación natural y canalizarla haciendo sentir a los estudiantes responsables de sacar adelante una idea.

Carolina le pide que oculten las circunferencias y Margarita le pide que arrastre los elementos libres de la construcción. Es una manera instituida en la clase para validar una construcción propuesta por una estudiante, como indicador de asumir la responsabilidad por la validación [E-APA ]. Ellas sugieren cómo intervenir sobre la representación antes de tomar una postura a favor o en contra. Como un mecanismo para impulsar la discusión por parte de los estudiantes, la profesora les pide observar qué sucede cuando se arrastran los puntos $A$ y $B$ o los extremos del segmento auxiliar [P-PCP $]$.

En un momento dado del arrastre, Margarita objeta la construcción, diciendo que no es válida: "No, porque el segmento no puede ser congruente a ninguno de los demás" [73]. Ella identifica el caso en el que el segmento auxiliar y el segmento $A B$ tienen la misma longitud. Es decir, 
elabora un argumento empírico para rechazar la construcción [E-APE $]$ ]. Carolina explica que ese caso no se presenta siempre y lo exceptúan $\left[{\mathrm{E}-T P_{2}}_{2}\right]$. Es decir, ponen como condición que el segmento auxiliar y el segmento $A B$ no tengan la misma longitud. Finalmente, la construcción se aprueba.

Para finalizar la sesión, la profesora pide a los estudiantes argumentar por qué los lados $A C$ y $B C$ del triángulo son congruentes. Ella insiste en preguntar por un argumento teórico para validar la afirmación de la congruencia de los lados AC y AC [P-PQA] . Se dirige a Carla, quien no ha intervenido en la sesión. Ella, en tono bajo, menciona que los segmentos son congruentes "porque las circunferencias hacen que sean congruentes" [80]. La profesora problematiza el punto de vista de Carla [P-PPV $]$ y promueve la escucha de ideas para favorecer el intercambio comunicativo [P-PEI] , pues algunos estudiantes hablan cuando Carla interviene, y ella les llama la atención. Adicionalmente, cuando ve a Carla en dificultades para elaborar una argumentación, pide a otro estudiante que la apoye [P-OEA] ], situación que no es censurada (Forman, 1996) y promueve la confrontación de posturas $\left[\mathrm{P}-\mathrm{PCP}_{2}\right]$. Por parte de los estudiantes, llama la atención la participación de Sonia: "Es que no habla duro. iHabla duro, Carla!" [82], contribuyendo a regular la norma para favorecer un clima de comunicación $\left[\mathrm{E}-\mathrm{RN}_{2}\right]$, y de Cristian, quien expone un argumento teórico, con base en la congruencia de radios de circunferencias congruentes para validar la congruencia de los lados $A C$ y $B C$ : "Porque ambas circunferencias tienen la misma medida... Son congruentes, porque ambas circunferencias tienen el mismo radio" [84] $\left[\mathrm{E}-\mathrm{APT}_{1}\right]$.

\section{Discusión}

El siguiente análisis de los códigos, narración por narración, nos permite caracterizar en qué se enfoca la construcción del ambiente de indagación:

La narración 1 muestra que la interacción se centra en la argumentación, como lo indican el uso de los cinco códigos de esa categoría, en las once intervenciones catalogadas. La profesora y un estudiante preguntan por qué y los estudiantes dan argumentos empíricos o teóricos. En particular, es notorio en esta narración, y a lo largo de las otras tres narraciones, que la pregunta por el porqué es constante en el discurso de la profesora. Este discurso se da tanto para impulsar la construcción de significado (Goos, 2004) como para validar las producciones (Quaranta \& Tarasow, 2004).

La narración 2 exhibe un aspecto clave de un ambiente de indagación: la toma de postura. Esta se ve reflejada en el uso de cuatro códigos de esa categoría en nueve intervenciones catalogadas (de las once). Bien sea 
proponiendo acciones, dando y explicando puntos de vista o refutando lo dicho por otros, gracias a la mediación de la profesora, los estudiantes participan de manera relevante. En esta narración también usamos el código E-APA, que corresponde a la categoría responsabilidad, aunque este indicador solo se vio en dos intervenciones.

En la narración 3, nos enfocamos en la confrontación de ideas de los estudiantes, estimulada por la profesora, quien impulsa la argumentación. Los estudiantes, por su parte, toman posturas, argumentan, proponen acciones para verificar una idea y contribuyen a regular normas para facilitar la comunicación. De las veintiséis intervenciones que codificamos en esta narración, cinco se encuentran en la categoría de confrontación. Para estimularla, la profesora problematiza los puntos de vista, promueve la confrontación de ideas, orienta y aclara el uso del lenguaje e impulsa a los estudiantes a validar sus posturas. Todas estas son acciones que ella promueve buscando que los estudiantes encuentren criterios que les permitan validar por sí mismos sus producciones y dirimir los desencuentros (Quaranta \& Tarasow, 2004).

La narración 4 ilustra el papel de la profesora, que cede la responsabilidad a los estudiantes en la construcción de conocimiento. De las catorce intervenciones que codificamos, cuatro tienen esa intención, principalmente porque la profesora admite que su postura sea objetada y porque más que informar media el intercambio comunicativo, impulsa la toma de postura, orienta la confrontación y el establecimiento de acuerdos y permite a los estudiantes explicitar sus puntos de vista y participar en las decisiones tomadas (Furinghetti et al., 2001).

Consideramos que las cuatro narraciones reflejan los esfuerzos de la profesora para que los estudiantes sean los protagonistas de la actividad matemática. No solo su gestión, sino los problemas propuestos y el apoyo en GeoGebra, permitieron a los estudiantes "ofrecer y recibir ayuda, compartir frustraciones y exponer sus propias ideas" (Forman, 1996, p. 122).

Dos códigos no se ilustran en las cuatro narraciones: la aclaración de términos empleados por algún miembro de la comunidad (P-AT) y la valoración de los aportes individuales para promover la expresión de ideas (P-VAI). Estos aparecen en otros fragmentos analizados, que, por dificultades de espacio, no incluimos en el artículo. Con respecto al primero, según Goos (2004), es importante que el profesor impulse el buen uso de la terminología y el lenguaje para exponer las ideas de forma clara. Con respecto al segundo, aunque no es evidente en una intervención explícita, esta acción marca la pauta de actuación de la profesora a lo largo de la secuencia de enseñanza. 
Los códigos emergentes, fruto del análisis, muestran que las acciones de estudiantes y profesores crean un ambiente de indagación. Es un comienzo, y hay muchas más acciones que el profesor puede promover. Pero consideramos que, en la medida en que la búsqueda de un ambiente de indagación se vuelva una práctica constante, profesor y estudiantes tendrán mejores formas de intervenir. Por ejemplo, solo muy ocasionalmente a lo largo de la secuencia observamos que los estudiantes se hicieran preguntas unos a otros o aportaran a la producción de otros. Tampoco vimos que la profesora hiciera un énfasis en dar espacio para errar, corregir, divagar o ensayar. Este énfasis es posible si no hay tanta presión de la dirección del colegio o de los padres de familia por cubrir los contenidos propuestos en la programación, asunto que los profesores experimentan día a día.

\section{Conclusiones}

La generación de un ambiente de indagación no es una tarea sencilla. A pesar de tener referentes teóricos sobre cómo hacerlo, es la práctica la que da la experticia. El experimento de diseño descrito muestra una posible ruta para atender las recomendaciones que se hacen hoy en día a los profesores de orientar la actividad matemática de los estudiantes, más que modelar frente a ellos y permitirles solamente ejercitarse en procedimientos y cálculos (Goos, 2004; Ministerio de Educación Nacional, 2006). Cabe aclarar que no pretendemos afirmar que esta sea la única ruta posible, pues el experimento de enseñanza es limitado a la acción de una profesora con sus estudiantes; aunque sí nos permite mostrar una opción factible de llevar a cabo.

La participación de la profesora en el experimento le permitió vivir un cambio de paradigma que ha movilizado su acción educativa a posteriori, en la clase de geometría. En lugar de ir a la clase para exponer un tema, lleva problemas a los estudiantes, les permite apoyarse en GeoGebra, estimula el trabajo en grupos y promueve el debate en las plenarias. Como se aprecia en el análisis y la discusión, el impulso a la formulación de argumentos, la toma de postura, la confrontación de ideas y ceder la responsabilidad en la construcción de conocimiento son aspectos que destacamos como respuesta al interrogante que movilizó el estudio. Sin embargo, gestionar la interacción discursiva entre los estudiantes, promover la expresión de ideas con claridad, asegurarse de que los estudiantes interpretan lo dicho por un compañero y promover la toma consensuada de decisiones, entre otros, son retos que la profesora deberá incorporar en su práctica cada vez de mejor manera.

Adicionalmente, uno de los compromisos que debe asumir la profesora, así como quienes deseen promover un ambiente de indagación, es 
evitar a toda costa valorar como "correcta" o "incorrecta" una idea de los estudiantes y cederles la responsabilidad de introducirse en la búsqueda de criterios para validar sus propias ideas, de modo que la profesora sea mediadora del establecimiento de acuerdos. Esto es necesario para que los estudiantes asuman un papel protagónico, propongan, tomen decisiones y vean la necesidad de escuchar y valorar los aportes de los compañeros.

Es importante resaltar que la profesora y los estudiantes iniciaron la conformación de una comunidad de práctica, y aunque ella sea la persona con más experiencia de todos los miembros, permite ser objetada. La actitud receptiva y de disposición de la profesora no hace que su conocimiento y experticia sea visto por los estudiantes como inferior, sino todo lo contrario. Además, le proporciona oportunidades para afianzar lo que sabe y mejorar su hacer.

No sobra señalar la influencia de las tareas propuestas a los estudiantes en la constitución de un ambiente de indagación. Al involucrar de forma activa a los estudiantes en acciones propias de la actividad matemática, tales como explorar, descubrir, conjeturar y justificar, impulsa a los estudiantes a comprometerse con su producción y a defenderla.

\section{Sobre las autoras}

Eliana Martínez-Mora es normalista superior, con énfasis en Educación Artística. Licenciada en Matemáticas, especialista en Educación Matemática y magíster en Docencia de las Matemáticas de la Universidad Pedagógica Nacional, Colombia.

Leonor Camargo-Uribe es profesora titular e investigadora en el área de Didáctica de las Matemáticas. Autora de libros y artículos de investigación sobre aprendizaje y enseñanza de la geometría, con énfasis particular en la línea de argumentación y prueba. Es líder del grupo de investigación Didáctica de la Matemática, clasificado en Colciencias.

\section{Referencias}

Camargo, L. \& Sandoval, I. (2017). Acceso equitativo al razonamiento científico mediante la tecnología. Revista Colombiana de Educación, 73, 179-211.

Cotton, T. (1998). Towards a Mathematics Education for Social Justice (tesis doctoral). Nottingham: University of Nottingham, Reino Unido.

Forman, E. (1996). Learning mathematics as participation in classroom practice: Implications of sociocultural theory for educational reform. En L. Steffe, P. Nesher, P. Cobb, G. Goldin \& B. Greer, Theories of Mathematical Learning (pp. 115-129). Mahwah, NJ: Lawrence Erlbaum. 
Furinghetti, F., Olivero, F. \& Paola, D. (2001). Students approaching proof through conjectures: Snapshots in classroom. International Journal of Mathematical Education in Science and Technology, 32(3), 319-335.

Goos, M. (2004). Learning mathematics in a classroom community on inquiry. Journal for Research in Mathematics Education, 35(4), 258-291.

Laborde, C. (2000). Dynamic geometry environments as a source of rich learning contexts for the complex activity of proving. Educational Studies in Mathematics, 44(1-3), 151-161.

Mariotti, M. A. (2000). Introduction to proof: The mediation of a dynamic software environment. Educational Studies in Mathematics, 44(1-3), 25-53.

Ministerio de Educación Nacional (MEN). (2006). Estándares básicos de competencias en matemáticas. Bogotá: Ministerio de Educación Nacional.

Molina, M., Castro, E., Molina, J. \& Castro, E. (2011). Un acercamiento a la investigación de diseño a través de los experimentos de enseñanza. Enseñanza de las Ciencias, 29(1), 75-88.

Puentes, J. (2015). Ambiente indagativo y argumentación en un contexto de geometría dinámica: una experiencia en grado séptimo (tesis de maestría). Universidad Pedagógica Nacional, Bogotá, Colombia.

Quaranta, M. \& Tarasow, P. (2004). Validación y producción de conocimientos sobre las interpretaciones numéricas. Relime, 7(3), 219-233.

Richards, J. (1991). Mathematical discussions. En E. von Glasersfeld (ed.), Radical Constructivism in Mathematics Education (pp. 13-51). Dordrecht: Kluwer Academic.

Samper, C. \& Molina, O. (2013). Geometría plana: un espacio de aprendizaje. Bogotá: Universidad Pedagógica Nacional.

Skovsmose, O. (2000). Escenarios de investigación. Revista EMA, 6(1), 3-26.

Yackel, E. \& Cobb, P. (1996). Sociomathematical norms, argumentation and autonomy in mathematics. Journal for Research in Mathematics Education, 27(4), 458-477.

Yevdokimov, O. (2005). About a constructivist approach for stimulating students' thinking to produce conjectures and their proving in active learning of geometry. Recuperado de https://eprints.usq.edu.au/3352/1/1-Yevdokimov_ CERME4.pdf 